\title{
Sharing Lived Experiences and Indigenous Epistemologies in the Townsville Aboriginal and Islander Teacher Education Program
}

\author{
Lyn Nichols
}

James Cook University

etropic 14.1 (2015): 44-49. http://www.reefandleaf.com.au/etropic.html \& http://www.jcu.edu.au/etropic

I respectfully acknowledge the Aboriginal and Torres Strait Islander Traditional Owners, past, present and future of our lands. In particular I acknowledge the Bindal and Wulgurabba peoples on whose lands the James Cook University in Townsville has been built and on whose lands generations of scholars have and will continue to walk the paths of their ancestors who have not only gone before us, but continue to walk in spirit among us today.

\begin{abstract}
The research described in this paper explores the experiences of both students and teachers in the Aboriginal and Islander Teacher Education Program (AITEP) that operated during the late 1970s to early 1990s in Townsville, Australia. Using a phenomenological research this study explores the perceptions of the program and its influence based upon the experiences of those who participated in it, both Indigenous and non-Indigenous and to identify what they believe were elements that supported them to success. Preliminary findings from the study are presented. Implications of these experiences to current considerations in pre-service Indigenous teacher education are also presented.
\end{abstract}

Keywords: Aboriginal and Torres Strait Islander teacher education; phenomenology

\section{Introduction}

AITEP (Aboriginal and Islander Teacher Education Program) commenced in 1977 in Townsville, North Queensland, was, and still is, regarded as a national and international success in Indigenous teacher education in Australia (Henderson, L. Personal Communication. 2011). The question that immediately arises from this assertion and informs this study is how one might define the 'success' of AITEP. More importantly, who defines it as successful? For AITEP to be considered a success, a variety of perspectives are necessary, especially from those of the Aboriginal and Torres Strait Islander participants of AITEP. In response to this, the central focus of this study is to explore perceptions of the program's and its influence based upon the experiences of those who participated in it, both Indigenous and non-Indigenous and to identify what participants believe were elements that supported their individual and the program's success. It is a study that explores the lived experiences of those who participated in the program. It gives authority to their voice, especially the AITEP students who were the first generation of Aboriginal and Torres Strait Islander families to have experienced Western tertiary teacher education. 


\section{Background to the Study}

AITEP (Aboriginal and Islander Teacher Education Program) was a part of a national teacher education strategy in the early 1980s to produce 1000 Australian Indigenous teacher by 1990. Although many Aboriginal and Torres Strait Islander peoples graduated from this program as teachers and developed successful careers in classroom teaching, this ambitious goal was never reached. Now, more than 30 years later, there is another national goal to produce more Aboriginal and Torres Strait Islander teachers for Australian classrooms. According to the 2012 Report into the retention and graduation of Aboriginal and Torres Strait Islander students enrolled in teacher education, the "More Aboriginal and Torres Strait Islander Teacher Initiative (MATSITI) is a four-year (2012-2015) program that aims to increase the number of Aboriginal and Torres Strait Islander people who both enter and remain within professional teaching positions in Australian schools. MATSITI is aligned with the Council of Australian Governments (COAG) National Indigenous Reform Agreement and associated Closing the Gap targets to address Aboriginal and Torres Strait Islander disadvantage, and constitutes a \$7.5 million initiative announced by the Commonwealth Minister for Schools, Early childhood and Youth" (University of South Australia, 2012). Therefore this research is deemed significant in identifying those program elements that support the success of Aboriginal and Torres Strait Islander people to become teachers.

\section{Focus of the Study}

Seeking an understanding of the contributing influences to such success was for me as both a researcher and a past AITEP student problematic. In addition to the Western ideals and knowledges deemed necessary for an Australian Indigenous tertiary education, this program consciously or unconsciously likely contained elements of significance for Indigenous student success. That is, factors inextricably linked to the Indigenous learners' cultural epistemologies were likely foundations for the formal and informal dynamics of the program. Further it is likely that these formal and informal elements were perceived differently by participants because of the diverse student population the program catered to. The Aboriginal and Torres Strait Islander students came from urban, regional, remote and community-based homes nation-wide to enrol in AITEP. As well, not only were there two generic groups consisting of Aboriginal and Torres Strait Islanders, there were a vast array of nations within the program that were representative of each diverse culture. For instance, the cultural practices and beliefs of the Eastern Torres Strait Island participants, such as Murray Island, may have been considerably different to those from the Western Torres Strait Islands. Additionally, the Aboriginal tribal nations of the East Coast of Australia had significant differences in cultural practices and beliefs to each other and to that of inland and interstate Aboriginal nations. It is unlikely that because of this diversity a uniform experience was witnessed. The diversity of perceptions would likely be highlighted through the diversity in student language, identity, homelands, cultural practices and especially those relative to their environmental conditions, connections within and between cultures and cultural nations. This diversity would have influenced individual perceptions of tertiary experiences, tertiary successes and professional proficiencies within the workforce. Therefore, an exploration of the diverse Indigenous epistemologies and the lived experiences of Aboriginal and Torres Strait Islander students represented by those who were enrolled within the James Cook University's AITEP is essential to this research enquiry. Such an understanding is important to informing 
current teacher education efforts that may seek to develop programs of study and support structures based upon an assumption of homogeneity amongst an Indigenous student participants.

In line with the effort to ensure the study informs current developments in Indigenous teacher education, the study provides opportunities to also explore the challenges Aboriginal and Torres Strait Islander AITEP students faced within AITEP and the challenges they encountered thereafter as successful graduates in their transition to the professional worlds. For this reason, the study investigates the program's influence on their preparation for professional practice, especially those related to classroom teaching. Further, it provides opportunity for participants to describe the influences on their journey through life, especially during their time as participants in AITEP. This consideration will provide insight into how Indigenous students perceive professional careers as a part of their personal, culturally located identity. The study also includes the voices of Indigenous families who supported their student family members. This inclusion is made because those that attended AITEP, especially those from remote communities, often did so in association with family.

Equally important to this research is the inclusion of the perceptions of the James Cook University staff who were employed as instructors within AITEP and those who were engaged as support workers with the students of AITEP. Their review of working at the cultural interface as non-Indigenous academics and coming to terms with interacting with Australia's Indigenous cultures in the tertiary education world is equally important to highlight. This research seeks to highlight those pedagogies utilized by this group that they considered as having contributed to the success of so many Aboriginal and Torres Strait Islander peoples. Based upon the experiences of both Indigenous and non-Indigenous participants, the study seeks to determine if the perceptions of AITEP and their engagement with AITEP align with those of the Aboriginal and Torres Strait Islander students who were enrolled in AITEP. All of these considerations have considerable merit in informing current efforts in teacher education.

\section{Methodology}

This study uses a phenomenological methodological approach. Phenomenology was developed by the German philosopher Edmund Husserl in the second half of the nineteenth century. Using AITEP as the phenomena, data gathered seek to understand the experiences of those involved in AITEP. The study involves phenomenological interviews with Aboriginal and Torres Strait Islander ex-AITEP students and uses means by which participants can tell 'their story'. NonIndigenous interview participants are also provided the option of 'telling their story' rather than giving their information through formal interviews. In contrast to Western data gathering methods, the Aboriginal and Torres Strait Islander participants are allowed to tell their story without interruption to the owner of the story - it is their story! To interrupt is to disempower the story teller from his/ her ownership of the story and the information in it or their perceptions about the event. As each ex-AITEP student who witnessed and participated in the same phenomenon tell their version of their experiences, the culmination of 'their stories' about the one phenomenon makes the phenomenon whole - it provides a representation of the whole picture. This 'whole picture' of the AITEP phenomenon then will also include accounts relevant to the Aboriginal and Torres Strait Islander lived experiences prior to, during and following their 
involvement in the AITEP. This account will likely include reference to Aboriginal and Torres Strait Islander pre-colonial cultural practices, colonization histories and assimilation into mainstream Australian society. Linda Tuhawai Smith (1999) suggests that "Indigenous research should include elements of healing, decolonization, spiritual and recovery” (p.116). Therefore, any research methodological practice that relies on or conjures up the recalling of past colonial and imperialistic injustices against Aboriginal and Torres Strait Islander peoples must incorporate processes of healing.

Phenomenological expression not only elicits thought, feelings, signs, images, ideas and messages, but for Aboriginal and Torres Strait Islander peoples it also involves the metaphysical beliefs and practices of their cultures that allow each to make sense of their past, expresses their present and gives directions and hope for their futures. It is the wholeness of their story. A small but significant element in this research is that not only is it being undertaken by an Australian Aboriginal researcher, this Aboriginal researcher was also a student of the Townsville AITEP during the early 1980s. Hence, there are two critical Aboriginal and Torres Strait Islander research principles operating within this study. First, the researcher who is from the same and a similar culture and can readily identify the cultural significance of meanings, nuances and language signifiers that are important to both the interview participants. Second, the researcher is not 'looking from the outside in' but holds a paramount position within the research phenomena.

\section{Preliminary Findings}

Interview data gathered through the phenomenological interviews to date suggest that there are similarities and differences in the perceptions of AITEP amongst participants. The two evident similarities between the non-Indigenous participants and the Aboriginal and Torres Strait Islander participant stories relate to (1) the perceived notions of AITEP being a successful program and (2) the elements of academic support that addressed the needs of the 'whole' student. This support includes reference to the social and economic status and issues that continually confronted the Aboriginal and Torres Strait Islander AITEP student participants.

A few preliminary differences are presented at this time. One of the differences in the participant's perceptions was in relation to the philosophy and goals of the AITEP. The nonIndigenous participants, who were the AITEP and James Cook University staff members, knew what was expected of the program and what outcomes the program sought to achieve in line with the national imperative for 'producing' more Indigenous teachers. In contrast, the Aboriginal and Torres Strait Islander participants had little awareness of this imperative for AITEP. As well, this imperative, if known, did not hold much importance to student participants. In terms of the program's outcomes, the students knew it was developed to promote graduation of Aboriginal and Torres Strait Islander teachers. Although there was a program imperative, participants' primary commentary pertains to personal imperatives. They sought teaching qualifications that would open doors to higher levels of employment and greater economic prospects, especially as members of a society that provided few opportunities for Indigenous professionals.

Another difference between the non-Indigenous and the Aboriginal and Torres Strait Islander perceptions about AITEP was in relation to the perceived reasons for the closing of the AITEP after only 15 years of operations, especially as it was considered to have been a successful 
program and the Canadian ITEPs, on which AITEP was based, were still operating. The perceptions for the closure of the program from non-Indigenous participants were based upon perceived justified reasons such as the lack of interest from the Aboriginal and Torres Strait Islander urban communities; higher Aboriginal and Torres Strait Islander enrolments in the sciences and social sciences; and the withdrawal of government funding. In contrast, the Aboriginal and Torres Strait Islander participants made little mention of reasons for the program ended and instead lamented the cessation of the program especially since no on-campus alternative enclave teacher education support being offered. Graduates talked strongly about the consequence of the program to their lives both personally and professionally. They believed that there were still Aboriginal and Torres Strait Islander peoples within the urban and regional communities that wanted to become teachers and would have benefitted greatly in terms of achieving success from AITEP or a similar on-campus enclave program.

A final perception differences relates to what strategies were regarded as the most successful in supporting Aboriginal and Torres Strait Islander trainee teachers. Non-Indigenous academic participants commonly suggested pedagogical support in terms of curricula content, resources, delivery and support as a vital contributor to student success. Non-Indigenous participants commonly identified the provision of social and economic support to Aboriginal and Torres Strait Islander students as a significant contributor. The social support included: transportation; marriage/partner counselling; prejudice and racism counselling; legal issues that included police harassment and bailing students out of jail and involvement in student, community and cultural events. Economically, AITEP staff supported students through many financial hardships. In contrast, the Aboriginal and Torres Strait Islander participants believed that the most significant successful strategy to achieve tertiary success came from within the student body. In other words, the peer, collegial and cultural support from each other was what helped them to overcome the obstacles of a foreign tertiary environment and to become successful graduates. One Aboriginal participant described overcoming the racism as a group as an ongoing experience in "debriefing and regrouping”. A Torres Strait Islander participant aptly described the cultural support from each other as, "We followed our old cultural ways and formed our own tribes and clans within AITEP based on each annual intake of students". For example, each annual cohort was a 'tribe' and the 'clans' would be based on the level of study students took such as primary or early childhood education. It would also seem that these AITEP tribes and clans had their own cultural nuances, including verbal and non-verbal communication for operating within lectures and the university.

Several of the Aboriginal and Torres Strait Islander interview participants believed they owed their success in part to their spiritual guides who watched over them. This came from the moments of remembrance to AITEP students who were now deceased, to their significant family members and Elders who had also passed on such as mothers and fathers and grandparents and, to the Aboriginal spiritual ancestors who still inhabited the lands on which the James Cook University stands and where the students studied. Many non-Indigenous researchers would have difficulty in comprehending Indigenous spirituality or believe that it is a profound component of Indigenous identity. From an Aboriginal point of view this could be seen as a blessing thereby protecting delicate Aboriginal knowledges and their stories from misinterpretation and misappropriation. 
Finally, both the non-Indigenous and Aboriginal and Torres Strait Islander participants agreed that AITEP was a successful program. The non-Indigenous participants alluded to the successes of individual students, particularly those who came from non-scholarly backgrounds, or labouring types of employment.. The most common, and strongly voiced, statement from the Aboriginal and Torres Strait Islander participants was “I wouldn't be where I am today without AITEP”.

\section{Conclusion}

The important intended outcome of this research is to make a positive contribution to the national efforts in teacher education, especially in the teaching and support of Aboriginal and Torres Strait Islander learners specifically at a tertiary level. Although there are large numbers of Aboriginal and Torres Strait Islander students enrolled in tertiary programs today compared to that of a decade or two ago, there are even greater numbers who today do not continue on to post-secondary studies or drop out of these programs. The James Cook University no longer promotes, engages and supports a cohort approach to engaging Aboriginal and Torres Strait Islander students in teacher education as it did with AITEP. Although currently there are support strategies for Aboriginal and Torres Strait Islander students within courses at the James Cook University, these are more inclined to be aimed at the individual student and not the group as a whole. Considering the importance of the supportive aspect of the cohort model, Aboriginal and Torres Strait Islander students no longer have the cultural peer support from each other as they did in the AITEP. This research considers this aspect of AITEP and Indigenous tertiary education to be important to both the attraction to teacher training and to the success of hundreds of Aboriginal and Torres Strait Islanders to becoming qualified teachers.

\section{Works Cited}

Byrne, M. M. (2001). Understanding life experiences through a phenomenological approach to research. In AORN Journal, 73 (4), 830-832, April, 2001.

Henderson, L. (2011). Personal communication. James Cook University. Townsville. Hughes, P., Willmott, E. (1982). 1979 NAEC Sponsored project report. The education and employment of Aboriginal and Torres Strait Islander Teachers. Retrieved: http://matsiti. edu.au/1000-teachers-by-1990.

Loos, N., \& Miller, G. (Eds.). (1987). Succeeding against the odds The Townsville Aboriginal and Islander Teacher Education Program. Allen and Unwin: Sydney.

Smith, L. T., (1999). Decolonizing Methodologies: Research and Indigenous Peoples. Zed Books. London.

University of South Australia (2012). More Aboriginal and Torres Strait Islander Teachers Initiative. MATSITI Project Plan 2012-15. David Unaipon College of Indigenous Education and Research. Version 1.2 | June 2012. www.matsiti.edu.au 